\title{
Field intercomparison of a novel optical sensor for formaldehyde quantification
}

\author{
Stephen Friedfeld, Matthew Fraser \\ Department of Environmental Science and Engineering, Rice University, Houston, Texas \\ David Lancaster, Darrin Leleux, Dirk Rehle, Frank Tittel \\ Department of Electrical and Computer Engineering, Rice University, Houston, Texas
}

\begin{abstract}
A one-week in situ intercomparison campaign was completed on the Rice University campus for measuring $\mathrm{HCHO}$ using three different techniques, including a novel optical sensor based on difference frequency generation (DFG) operating at room temperature. Two chemical derivatization methods, 2,4-dinitrophenylhydrazine (DNPH) and $o$ (2,3,4,5,6-pentafluorobenzyl)hydroxylamine (PFBHA), were deployed during the daylight hours for three- to four-hour time-integrated samples. A real-time optical sensor based on laser absorption spectroscopy was operated simultaneously, including nighttime hours. This tunable spectroscopic source based on difference frequency mixing of two fiberamplified diode lasers in periodically poled $\mathrm{LiNbO}_{3}(\mathrm{PPLN})$ was operated at $3.5315 \mu \mathrm{m}\left(2831.64 \mathrm{~cm}^{-1}\right)$ to access a strong $\mathrm{HCHO}$ ro-vibrational transition free of interferences from other species. The results showed a bias of -1.7 and -1.2 ppbv and a gross error of 2.6 and $1.5 \mathrm{ppbv}$ for DNPH and PFBHA measurements, respectively, compared with DFG measurements. These results validate the DFG sensor for time-resolved measurements of HCHO in urban areas.
\end{abstract}

\section{Introduction}

Accurate measurements of formaldehyde (HCHO) concentrations are important for a complete understanding of the basic chemistry that occurs in the atmosphere. Formaldehyde, directly emitted from primary sources and formed by the atmospheric oxidation of anthropogenic and biogenic hydrocarbon emissions, is readily photolyzed in the atmosphere leading to radical formation and elevated groundlevel ozone concentrations [National Research Council, 1991].

Typical concentrations of $\mathrm{HCHO}$ range from sub-ppbv (parts per billion volume) levels in remote and rural areas to higher levels (up to 10-20 ppbv) in urban atmospheres [Carlier et al., 1986; Lee et al., 1995]. Chemical derivatization methods have long been used for HCHO detection. However, these techniques are limited by the required time integration of sample collection, which does not describe the rapid fluctuations in HCHO concentrations. The scale of these fluctuations is important as $\mathrm{HCHO}$ is an intermediate in the photooxidation of hydrocarbons that undergoes rapid photolysis to produce $\mathrm{HO}_{\mathrm{x}}$ radicals [Fried et al., 1998]. Despite the advantages of $\mathrm{HCHO}$ derivatization such as simplicity and relatively low cost, spectroscopic techniques provide a

Copyright 2000 by the American Geophysical Union.

Paper number 2000GL011573.

0094-8276/00/2000GL011573\$05.00 reliable and reproducible means of time-resolved collection of atmospheric HCHO concentrations [Vairavamurthy et al., 1992; Fried et al., 1998].

Several intercomparison studies have monitored HCHO formation and degradation [Trapp and de Serves, 1995; Gilpin et al., 1990; Apel et al., 1998]. These studies compared spectroscopic techniques, such as tunable diode laser absorption spectroscopy (TDLAS), Fourier transform infrared spectroscopy (FTIR), and differential optical absorption spectroscopy (DOAS), to wet chemical techniques (both near-real time and time-integrating) usually based on derivatization with 2,4-dinitrophenylhydrazine (DNPH) [Tanner et al., 1996]. However, advances in nonlinear optical materials have allowed a novel optical sensor, based on difference frequency generation (DFG), to be developed for high quality $\mathrm{HCHO}$ concentration measurements that do not require cryogenic cooling. Building upon previous efforts [Mine et al., 1997; Lancaster et al., 1998; Richter et al., 1998; Lancaster et al., 1999], the DFG based sensor can now provide real-time detection of atmospheric $\mathrm{HCHO}$ with a minimum detectable sensitivity of approximately $0.5 \mathrm{ppbV}$ [Lancaster et al., 2000]. The wavelength selectivity of the DFG method results in less susceptibility to interference from water vapor.

In this paper, we report on results from an in situ intercomparison between two derivatization methods, DNPH and $o$-(2,3,4,5,6-pentafluorobenzyl)hydroxylamine (PFBHA), and a DFG-based gas sensor for the collection of atmospheric $\mathrm{HCHO}$. This represents the first field intercomparison of a DFG sensor which promises reliable and robust $\mathrm{HCHO}$ quantification using a real-time, autonomous diode laser based sensor [Lancaster et al., 2000], with well established wet chemical trace gas detection techniques. The results of this intercomparison show the DFG precision to be roughly equal to the bias between simultaneous DFG and timeintegrating wet chemical $\mathrm{HCHO}$ measurements, validating the use of a DFG sensor for quantitative measurements of $\mathrm{HCHO}$ in urban areas.

\section{Experimental}

Site. The intercomparison campaign occurred from November 29 to December 6, 1999 on a rooftop at an elevation of 20 meters on the Rice University campus. During sampling, temperature and humidity varied from 5-25C and $65-95 \% \mathrm{RH}$ and included a period of precipitation. The campus is situated in central Houston, where vehicular and industrial emissions of HCHO mix with biogenic hydrocarbons emitted from local parks and treed neighborhoods so that both primary and secondary sources of HCHO might 


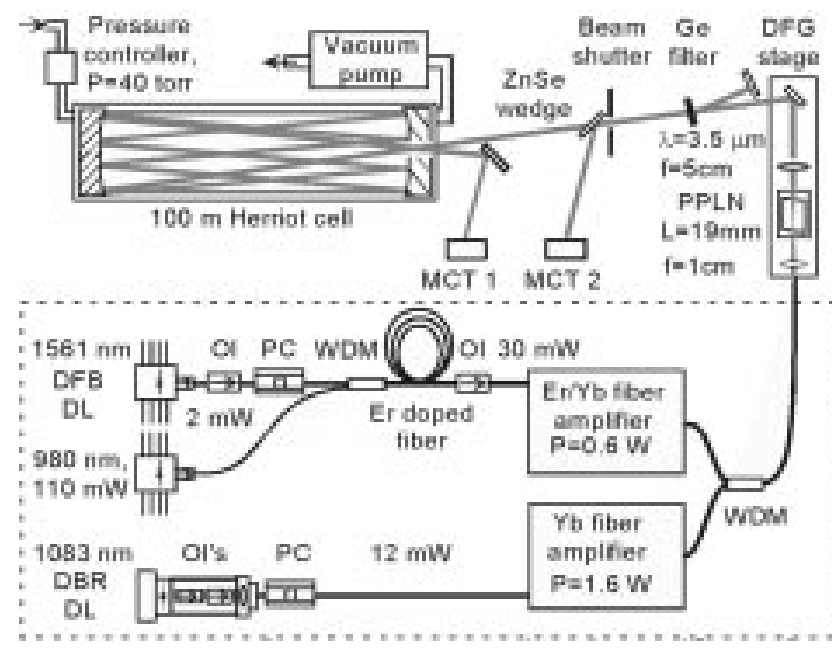

Figure 1. Schematic of DFG based gas sensor configured for HCHO detection: DFB, distributed feedback; DL, diode laser; DBR, Distributed Bragg; OI, opto-isolators; PC, polarization controller.

be important. The rooftop location allowed free airflow in all directions so air sampled represented the nearby region rather than localized sources.

\section{Derivatization Methods}

DNPH and PFBHA. In the DNPH method, Waters $\mathrm{SepPakC}_{18}$ cartridges were coated with a DNPH solution consisting of twice-recrystallized pure DNPH and the highest grade $5 \mathrm{M} \mathrm{HCl}$ and acetonitrile. Air was sampled at a rate of 1 liter $\min ^{-1}$ for typically a period of 3 to 4 hours using SKC Airchek Sampler pumps which were calibrated both before and after the experiment. For each sample, a field blank was also taken simultaneously at the site. The cartridges were extracted with acetonitrile, and the resultant HCHO hydrazones were analyzed with liquid chromatography (LC) followed by UV detection using a Hewlett Packard 1100 LC [Fung and Grosjean, 1981]. The HPLC instrument response was calibrated using commercially available standards.

For the PFBHA method, air is pulled into a glass impinger containing $15 \mathrm{~mL}$ deionized water at a flow rate of 1 $1 \mathrm{~min}^{-1}$. Upon sampling completion, an acidic solution of PFBHA is added, and the resultant oxime is extracted into hexane and subsequently analyzed with gas chromatography/mass spectrometry using a Hewlett Packard 5973 Mass Sensitive Detector [ $Y u$ et al., 1995].

Diode Laser-based Method. For highly selective optical quantification of $\mathrm{HCHO}$, a fundamental ro-vibrational absorption line near $2831.64 \mathrm{~cm}^{-1}(3.5315 \mu \mathrm{m})$ free from potential interfering background gases $\left(\mathrm{H}_{2} \mathrm{O}, \mathrm{CH}_{4}\right)$ was monitored using a mid-infrared DFG based spectroscopic source. Since the DFG system requires only Peltier cooling, no consumables such as liquid nitrogen are needed. This offers the potential for long term remote gas monitoring and control using a modem connection. A schematic of the DFG sensor configuration is shown in Figure 1. The two diode seed lasers are a fiber-coupled single frequency $2 \mathrm{~mW}, 1561 \mathrm{~nm}$ DFB diode laser and a $50 \mathrm{~mW}, 1083 \mathrm{~nm}$ DBR diode laser. An $\mathrm{Er}^{3+}$ doped fiber amplifier increases the $1561 \mathrm{~nm}$ seed power to $30 \mathrm{~mW}$ to saturate the gain in the $0.6 \mathrm{~W} \mathrm{Er} / \mathrm{Yb}$ fiber amplifier while the $1083 \mathrm{~nm}$ diode directly seeds 1.6 W Yb amplifier [Goldberg et al., 1999]. To combine the two DFG pump channels into a single fiber, a wavelength division multiplexer (WDM) is used and the two pump beams are imaged by a $1 \mathrm{~cm}$ focal-length achromatic lens into the $19 \mathrm{~mm}$ long periodically poled lithium niobate (PPLN) crystal with grating periods ranging from 29.7 to $30.5 \mu \mathrm{m}$. A 5 $\mathrm{cm}$ focal length $\mathrm{CaF}_{2}$ lens then collects the tunable, narrowband $(<60 \mathrm{MHz})$ radiation generated in the PPLN crystal. While up to $0.7 \mathrm{~mW}$ of mid-infrared DFG power has been generated previously, this work used $0.1 \mathrm{~mW}$ [Lancaster et al., 1999], sufficient power for a balanced detection scheme. The collimated DFG beam is then divided into two beams by a $\mathrm{ZnSe}$ wedge. The primary beam is directed through an astigmatic Herriot multipass absorption cell, configured for a path length of $100 \mathrm{~m}$ to a low noise $\mathrm{HgCdTe}$ (MCT) detector. The remaining reference beam is incident onto a second MCT detector. Such a dual beam absorption technique leads to a significant reduction of optical noise due to interference effects by the various optical components in the beam path, which is eliminated by ratioing the signals from both MCT detectors. The MCT detectors were operated in a photoconductive mode and DC coupled to preamplifiers with $3-\mathrm{db}$ bandwidth of $200 \mathrm{kHz}$. The data from the spectroscopic measurements were acquired simultaneously by the use of two analog-digital data acquisition cards and interfaced to a laptop computer. Spectral lines were acquired by direct current modulation of the $1561 \mathrm{~nm}$ pump diode laser (triangular waveform modulation at 0.1 to $1 \mathrm{kHz}$ ). Although determination of absolute concentrations is an inherent feature of direct absorption spectroscopy, the DFG sensor calibration was confirmed prior to HCHO concentration measurements in ambient air. For this purpose a certified mixture of $\mathrm{HCHO}$ (760 ppbv) in $\mathrm{N}_{2}$ from Scott Speciality Gases was used. Lower concentrations down to 20 ppbV with accuracy of $10 \%$ were produced using $\mathrm{N}_{2}$ dilution by means of a dual mass flow controller based gas mixing system. Measured HCHO concentrations were in good agreement with the mixed calibration gas composition.

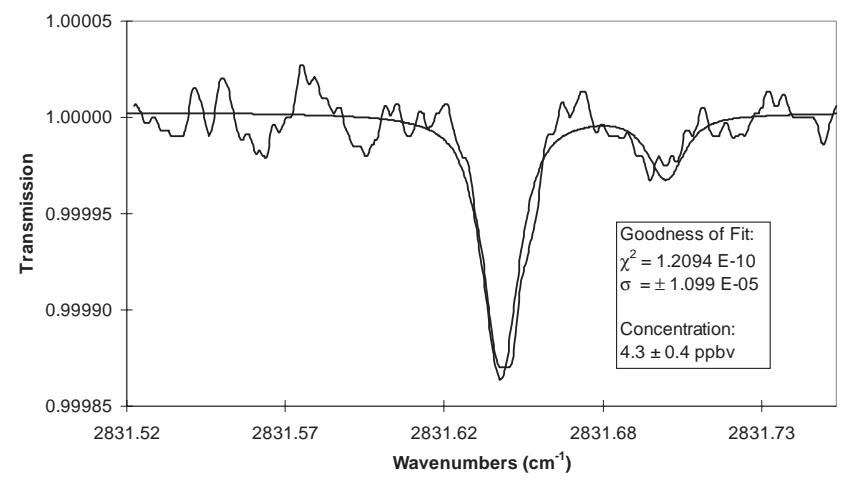

Figure 2. Spectrum of atmospheric HCHO at $2831.6417 \mathrm{~cm}^{-1}$ $(3.53 \mu \mathrm{m})$ in a multipass cell with an effective optical path length of $100 \mathrm{~m}$ at $\mathrm{P}=40$ Torr. This measurement was taken over 5000 averages at a $100 \mathrm{~Hz}$ scan rate. 

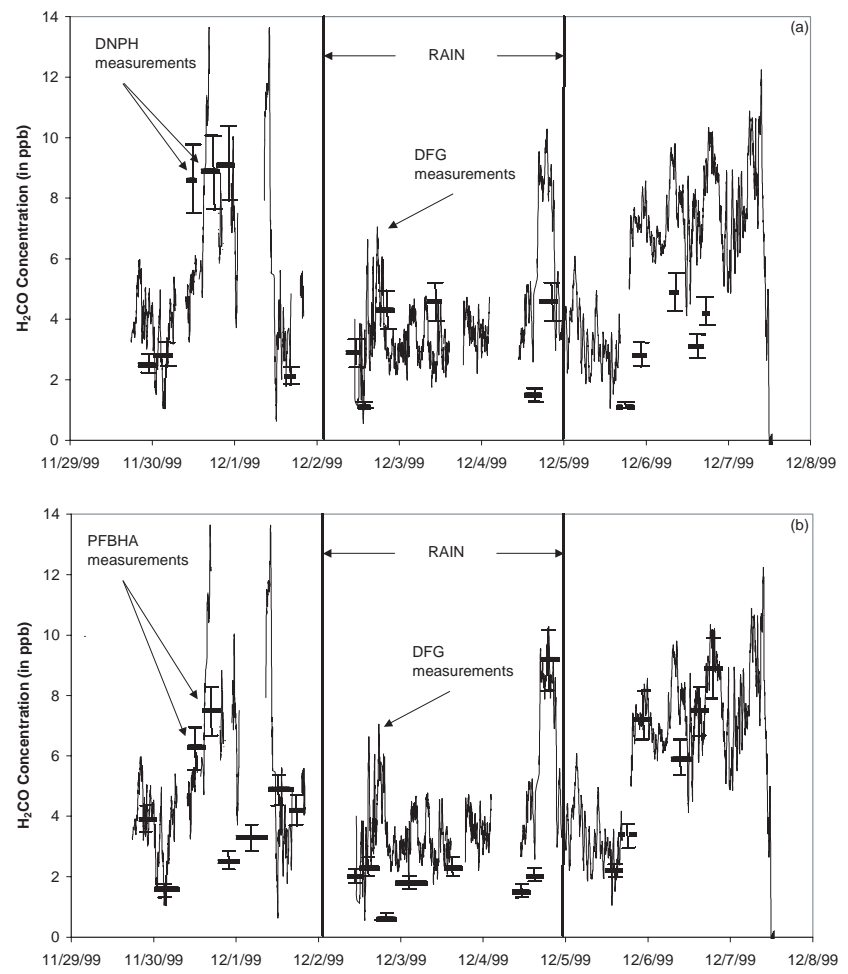

Figure 3. Intercomparison of atmospheric $\mathrm{HCHO}$ concentration measurements for a 9 day period from November 29 to December 7, 1999 by three independent trace gas detection techniques in Houston, TX. Several brief gaps in the DFG based measurements are apparent. These occurred as a result of unscheduled power interruptions and scheduled sensor performance verification.

\section{DFG Measurements}

The DFG sensor acquired continuous on-line concentration data for $52 \mathrm{~s}$ sampling periods. The optimum $\mathrm{HCHO}$ sampling pressure is a compromise between absorption sensitivity (best at high pressure) and line selectivity (best at low pressure). As the sampling pressure is reduced, the detection sensitivity does not decrease below the value at atmospheric pressure until a point at which Lorentzian and Doppler linewidths are equal (which typically occurs between 10 and 50 torr). This is the pressure regime at which this HCHO sensor was operated (i.e., 40 torr). In this pressure range the lineshape can be described as a convolution of Lorentzian and Doppler lineshapes known as a Voigt profile.

The particular molecular transition of interest occurs at $2831.6417 \mathrm{~cm}^{-1}$. This line is free from interference of other transitions as mentioned above and shows a weak satellite transition at $2831.6987 \mathrm{~cm}^{-1}$ [Fried et al., 1998]. This smaller peak provides additional information that can be used to improve precision and accuracy of HCHO concentration measurements. Since the distance between the peaks as well as their locations are given by the HITRAN spectroscopic data base, the frequency per point in the algorithm can be calculated. The line intensity of both peaks as well as their ratio, and Voigt linewidth of $1.436 \times 10^{-2} \mathrm{~cm}^{-1}$ (FWHM), are also known from HITRAN.

A representative $\mathrm{HCHO}$ concentration measurement of ambient air obtained with the $100 \mathrm{~m}$ long multipass cell at a total pressure of 40 torr is shown in Figure 2. An average of 5000 spectra were acquired in a time interval of $140 \mathrm{~s}$ for each concentration measurement. The DFG based sensor employs real time data analysis based on Labview software and no further computer processing is required. A dualVoigt profile is fitted using a nonlinear least squares technique known as the Levenberg-Marquardt algorithm and is overlaid on the experimentally obtained spectrum indicating a concentration value of $4.3 \mathrm{ppbV} \mathrm{HCHO}$. The residual between the data and the Voigt line fit displays a standard deviation of $\pm 1.1 \times 10^{-5}$, corresponding to a concentration of $0.4 \mathrm{ppbV}$, a precision limit of the current instrument.

\section{Results and Discussion}

The HCHO concentration data are plotted in Figure 3 using a 9-point rolling average for the DFG measurements. The data are divided into three time segments, depicted by vertical lines in the figure. There was no precipitation in the first and third segments; in the second segment, $25.8 \mathrm{~cm}$ rain fell during 36 hours. Furthermore, the divisions also coincide with three sets of DNPH-impregnated cartridges, which were freshly prepared at the beginning of each 2-day segment to minimize potential contamination through excessive storage.

The horizontal bars of Figures $3 \mathrm{a}$ and $3 \mathrm{~b}$ represent the time-integrated average $\mathrm{HCHO}$ concentrations measured using the DNPH and PFBHA methods, respectively. The DNPH results were calculated by subtracting the overall average blank concentration from each sample concentration, as determined from HPLC-detector peak area. HCHO levels quantified from field blanks were, on average, approximately $30 \%$ of $\mathrm{HCHO}$ quantified from collected samples. This average field blank level is equivalent to an atmospheric $\mathrm{HCHO}$ concentration of $1.2 \mathrm{ppbV}$. The variability of the field blanks is significant with a standard deviation equivalent to an atmospheric HCHO concentration of $0.9 \mathrm{ppbv}$, and is a limit to the sensitivity of the DNPH method. This is a common shortcoming of the DNPH method [Sirju et al., 1995]. Error bars for the DNPH and PFBHA measurements are given in the figure for the estimated precision of the measurements and were determined separately by parallel sampling for three-hour periods for both derivatization methods.

The bias and gross error between the DNPH measurements and the DFG measurements and the PFBHA and DFG measurements were calculated. Bias is defined as the mean residual concentration $\left(\mathrm{C}_{\mathrm{DNPH}}-\mathrm{C}_{\mathrm{DFG}}\right.$ or $\mathrm{C}_{\mathrm{PFBHA}}-$ $\mathrm{C}_{\mathrm{DFG}}$ ) based on a comparison of all individual observations, while absolute gross error is the mean absolute value of the residual concentrations. A cut-off value of $0.1 \mathrm{ppbv}$ was used, where measurements below 0.1 ppbv were excluded from the calculation. The bias and gross error between HCHO concentrations from DNPH and DFG methods were -1.7 ppbv and $2.6 \mathrm{ppbv}$, resulting in a normalized bias and normalized gross error of $-24 \%$ and $49 \%$, respectively. For the comparison of the PFBHA and DFG methods, bias and gross error were $-1.2 \mathrm{ppbv}$ and $1.5 \mathrm{ppbv}$; normalized bias and gross error were $-23 \%$ and $30 \%$.

Although the normalized bias between the DNPH and PFBHA derivatizations relative to the DFG methods are similar, the trends between the measurements are distinct. Significant differences between the DNPH and DFG measurements occur during the final stage of sampling. One possible explanation is the observed increasing variability in the DNPH field blank levels leading to inaccurate DNPH measurements. 
Time-resolved data measurements indicate the daily fluctuations in HCHO concentration in Houston. Though the time-integrated methods indicate general trends over a relatively long period of time (3 to 4 hours) compared to per minute measurements from DFG spectroscopy, the often sudden shifts in HCHO levels are not apparent with time integration. For example, the last three reported $\mathrm{HCHO}$ concentrations in segment 1 (Figure $3 \mathrm{~b}$, PFBHA vs. DFG) are $3.3,4.9$, and $4.2 \mathrm{ppbv}$, respectively. The per minute DFG measurements, however, reveal over six peaks and valleys during the same sampling time, with concentrations ranging from around $0.5 \mathrm{ppbv}$ to almost $14.0 \mathrm{ppbv}$. Similar observations exist for all time segments and with both derivatization methods compared to DFG measurements. Monitoring HCHO fluctuations, along with simultaneous time-resolved measurements of parameters such as ozone and oxides of nitrogen, is key to understanding the complexity of atmospheric reactions and for predicting atmospheric chemistry.

\section{Conclusions}

This work demonstrates the ability to perform on-line high quality $\mathrm{HCHO}$ concentration measurements without cryogenic cooling using optical absorption using difference frequency generation (DFG). Concentrations of $\mathrm{HCHO}$ between 1 and $15 \mathrm{ppbV}$ were detected using the DFG system with $0.5 \mathrm{ppbV}$ precision. This precision limit is similar to the bias calculated between DFG measurements and timeintegrating wet chemical quantifiations and validates the use of the DFG system for HCHO measurements in urban environments. Greater precision may be achieved in the future using wavelength modulation spectroscopy and longer optical pathlength to enable remote location $\mathrm{HCHO}$ quantification.

Acknowledgments. This work was funded by the Gulf Coast Hazardous Substance Research Center, the Texas Advanced Technology Program, NASA, the Welch Foundation and the National Science Foundation.

\section{References}

Apel, E., J. Calvert, D. Riemer, W. Pos, R. Zika, T. Kleindienst, W. Lonneman, K. Fung, E. Fujita, P. Shepson, T. Starn, and P. Roberts, Measurements comparison of oxygenated volatile organic compounds at a rural site during the 1995 SOS Nashville Intensive, J. Geophys. Res., 103, 22295-22316, 1998.

Carlier, P., H. Hannachi, and G. Mouvier, The chemistry of carbonyl compounds in the atmosphere-a review, Atmos. Environ., 20, 2079-2099, 1986.

Fried, A., B. Henry, B. Wert, S. Sewell, and J. Drummond, Laboratory, ground-based, and airborne tunable diode laser systems: performance characteristics and applications in atmospheric studies, Appl. Phys. B, 67, 317-330, 1998.

Fung, K. and D. Grosjean, Determination of nanogram amounts of carbonyls as 2,4-dinitrophenylhydrazones by highperformance liquid chromatography, Anal. Chem., 53, 168$171,1981$.

Gilpin, T., E. Apel, A. Fried, B. Wert, J. Calvert, Z. Genfa, P. Dasgupta, J. Harder, B. Heikes, B. Hopkins, H. Westberg, T. Kleindienst, Y.-N. Lee, X. Zhou, W. Lonneman, and S. Sewell, Intercomparison of six ambient [CH2O] measurement techniques, J. Geophys. Res., 102, 21161-21188, 1990.
Goldberg, L., J. Kaplow, and D. Kliner, Highly efficient 4-W Ybdoped fiber amplifier pumped by a broad-stripe laser diode, Opt. Lett., 24, 673, 1999.

Lancaster, D., D. Richter, R. Curl, and F. Tittel, Real-time measurements of trace gases using a compact difference frequency based sensor operating at $3.5 \mathrm{~mm}$, Appl. Phys. B, 67, 339-345, 1998.

Lancaster, D., D. Richter, R. Curl, and F. Tittel, High power continuous-wave mid-infrared radiation generated by difference frequency mixing of diode-laser seeded fiber amplifiers and its application to dual-beam spectroscopy, Opt. Lett., 24, 17441746, 1999.

Lancaster, D., A. Fried, B. Wert, B. Henry, and F. Tittel, Difference frequency based tunable absorption spectrometer for detection of atmospheric formaldehyde, submitted to Appl. Opt., May 2000.

Lee, Y.-N., X. Zhou, and K. Hallock, Atmospheric carbonyl compounds at a rural southeastern United States site, J. Geophys. Res., 199, 25933-25944, 1995.

Mine, Y., N. Melander, D. Richter, D. Lancaster, K. Petrov, R. Curl, and F. Tittel, Detection of formaldehyde using midinfrared difference-frequency generation, Appl. Phys. B, 65, 771-774, 1997.

National Research Council Rethinking the Ozone Problem in Urban and Regional Air Pollution, 500 pp., National Academy Press, Washington, DC, 1991.

Richter, D., D. Lancaster, R. Curl, and F. Tittel, Compact midinfrared trace gas sensor based on difference-frequency generation of two diode lasers in periodically poled $\mathrm{LiNbO}_{3}, A p p l$. Optics B, 67, 347-350, 1998.

Sirju, A.-P. and P. Shepson, Laboratory and field investigation of the DNPH cartridge technique for the measurement of atmospheric carbonyl compounds, Environ. Sci. Technol., 29, 384-392, 1995.

Tanner, R., B. Zielinska, E. Uberna, G. Harshfield, and A. McNichol, Concentrations of carbonyl compounds and the carbon isotopy of formaldehyde at a coastal site in Nova Scotia during the NARE summer intensive, J. Geophys. Res., 101, 2896128970, 1996.

Trapp, D. and C. de Serves, Intercomparison of formaldehyde measurements in the tropical atmosphere, Atmos. Environ., 29, 3239-3243, 1995.

Vairavamurthy, A., J. Roberts, and L. Newman, Methods for determination of low molecular weight carbonyl compounds in the atmosphere: a review, Atmos. Environ., 26, 1965-1993, 1992.

Yu, J., H. Jeffries, and R. LeLacheur, Identifying airborne carbonyl compounds in isoprene atmospheric photooxidation products by their PFBHA oximes using gas chromatography/ion trap mass spectrometry, Environ. Sci. Technol., 29, 1923-1932, 1995.

M. Fraser and S. Friedfeld, Department of Environmental Science and Engineering, Rice University, Houston, TX 77005. (email: mpf@rice.edu; sjf@rice.edu)

D. Lancaster, D. Leleux, D. Rehle, and F. Tittel, Department of Electrical and Computer Engineering, Rice University, Houston, TX 77005. (e-mail: davelanc@rice.edu, leleux@rice.edu, rehle@rice.edu, fkt@rice.edu)

(Received February 22, 2000; revised March 31, 2000; accepted May 16, 2000.) 